\title{
Idiopathic pulmonary fibrosis: unmasking cryptogenic environmental factors
}

\author{
Coralynn Sack ${ }^{1,2,3}$ and Ganesh Raghu ${ }^{1,2}$
}

Affiliations:

${ }^{1}$ Dept of Medicine, University of Washington, Seattle, WA, USA.

${ }^{2}$ Center for Interstitial Lung Disease, University of Washington, Seattle, WA, USA.

${ }^{3}$ Dept of Environmental and Occupational Health, University of Washington, Seattle, WA, USA.

Correspondence:

Ganesh Raghu, Center for Interstitial Lung Disease, University of Washington, Seattle, WA 98195, USA.

E-mail: graghuduw.edu

@ERSpublications

Occult environmental exposures are important factors in the pathogenesis of idiopathic pulmonary fibrosis. Research is needed to determine if eliminating these exposures prevents onset and/or disease progression in genetically susceptible persons. http://ow.ly/agd830mNGVU

Cite this article as: Sack C, Raghu G. Idiopathic pulmonary fibrosis: unmasking cryptogenic environmental factors. Eur Respir J 2019; 53: 1801699 [https://doi.org/10.1183/13993003.01699-2018].

ABSTRACT Idiopathic pulmonary fibrosis (IPF) is a progressive fibrotic lung disease of unknown origin that is associated with high morbidity and mortality. In this perspective, we briefly review the current understanding of the pathophysiology of IPF and the importance of environmental triggers as a precipitant of disease. We discuss occult intrinsic and extrinsic environmental factors that affect the lung microenvironment and may contribute to the development and progression of disease. The clinical implications of this framework need to be further elucidated, because prompt identification and elimination of occult exposures may represent a novel treatment modality. 


\section{Introduction}

Among the large, heterogeneous and complex family of interstitial lung diseases (ILD), idiopathic pulmonary fibrosis (IPF) has emerged as a clinically distinct entity associated with progressive fibrosis and a relatively poor prognosis. IPF is usually diagnosed in older adults, in their sixth or seventh decade, who present with insidious onset of dyspnoea, with or without cough, and bibasilar crackles. Disease is limited to the lungs without extra-pulmonary manifestations. High resolution computed tomography (HRCT) imaging of the chest characteristically shows subpleural fibrosis with honeycombing; histopathology demonstrates usual interstitial pneumonia (UIP) with fibroblastic foci interspersed with normal lung tissue. The treatment of IPF has recently focused on targeting the fibrotic pathway, an aftermath of onset of the disease pathway. The two available anti-fibrotic agents, pirfenidone and nintedanib, have only a modest effect on slowing the decline of forced vital capacity (FVC), a marker of disease progression, and on overall mortality.

One of the distinctive features of IPF is the lack of an apparent aetiology. Current diagnostic criteria require the exclusion of secondary causes of ILD, including domestic and occupational exposures, connective tissue disease and drug toxicity [1-3]. However, numerous studies have shown that environmental risk factors, including viral infections, microaspiration, tobacco smoke, air pollution and occupational exposures, are associated with disease pathogenesis (table 1). It is apparent that exposure to environmental factors alone is not sufficient to cause disease, but such factors may increase the probability of developing IPF in a genetically susceptible individual [4].

Many of the risk factors that are associated with IPF are also implicated in the pathogenesis of other ILDs. This observation has several possible explanations. First, many cases of IPF may be misdiagnosed as such and may simply represent manifestations of advanced stage ILDs with defined environmental causes. Second, similar environmental exposures may fuel ongoing inflammation and fibrosis in the lung, regardless of disease subtype. Third, IPF may represent an overlapping spectrum of ILDs that share a final common pathway of fibrosis [5].

TABLE 1 Overt or occult environmental risk factors for idiopathic pulmonary fibrosis

\section{Risk factor}

$\begin{array}{ll}\text { Intrinsic factors } & \text { Gastro-oesophageal reflux } \\ & \text { Microaspiration } \\ & \text { Microbiome } \\ & \text { Viral infection } \\ \text { Extrinsic factors } & \text { Tobacco smoke } \\ \text { Domestic/environmental } & \text { Wood fires } \\ & \text { Birds (including poultry, bird droppings, birdfeeders) } \\ & \text { Feather products (including feather duvets, comforter, pillows, jackets) } \\ & \text { Moulds (visible or unseen) } \\ & \text { Organic dusts } \\ & \text { Ventilation } \\ & \text { Hairspray } \\ & \text { Air pollution } \\ & \text { Welding } \\ & \text { Farming/agriculture } \\ & \text { Hairdressing } \\ & \text { Dentists/dental technicians } \\ & \text { Metal dust } \\ & \text { Wood dust/paper mill factory workers } \\ \text { Livestock, particularly birds } & \text { Nuclear waste/radiation hazards } \\ \text { Chemicals } \\ \text { Aluminium, Corion } ® \\ \text { Stone cutting/sand/granite/silica } \\ \text { Talc }\end{array}$

\#: these and other occupational exposures may be the cause of "occupational lung diseases" and thus not truly risk factors for patients diagnosed with idiopathic pulmonary fibrosis (IPF). Nevertheless, these may be occult if the patient otherwise diagnosed with IPF is not gainfully engaged with such environmental factors/exposures. 
In this perspective, we briefly review the current understanding of the pathophysiology of IPF and suggest that IPF should be regarded as an "environmental" disorder manifesting in genetically susceptible individuals. We discuss intrinsic and extrinsic environmental factors that affect the lung microenvironment and may contribute to the development and progression of disease. We also explore the potential clinical implications of this framework, and propose a new treatment strategy for IPF that attempts to prevent the onset or abort ongoing fibrosis by identifying and eliminating occult environmental exposures.

\section{Diagnosing idiopathic pulmonary fibrosis is not straightforward}

The diagnosis of IPF is inherently challenging, particularly in the setting of evolving diagnostic criteria and diverse clinical practices. The classification scheme of the idiopathic ILDs has gone through revisions, and IPF did not emerge as a distinctive entity with specific clinical, radiographic and histopathological findings until consensus guidelines were published in 2000 [6]. Even with adherence to the 2011 diagnostic criteria, misdiagnosis of the disease is common [7]. Consequently, patients determined as having IPF both in the past and at present may be best interpreted as having a spectrum of overlapping ILDs.

Current recommendations for making an accurate diagnosis of IPF emphasise the importance of multidisciplinary discussions among experts, including experienced pulmonologists, radiologists and pathologists (and rheumatologists on a case-by-case basis) [3]. The key radiographic and histopathologic features of the pattern of UIP have recently been refined $[3,8]$. In the appropriate clinical setting, the presence of UIP or probable UIP patterns on HRCT is sufficient to make a definitive diagnosis of IPF without surgical lung biopsy. Because some degree of uncertainty is often present, however, diagnosis frequently requires the integration of clinical, imaging and histologic information [3].

Many of the epidemiologic studies identifying risk factors for IPF were conducted in the 1990s and earlier, before current diagnostic criteria and advanced imaging technology were available. While UIP has been a recognised histologic pattern since the 1950s, the majority of patients involved in these studies did not undergo lung biopsy. Given the changing diagnostic criteria and likelihood of substantial disease misclassification, the results of epidemiologic studies must be interpreted cautiously.

The clinical history and exclusion of secondary causes of ILD are important, because a UIP pattern can be seen in many other fibrotic lung diseases, including connective tissue disease, asbestosis, hypersensitivity pneumonitis and sarcoidosis [9]. Identifying relevant pulmonary exposures requires a high index of suspicion, and failure to elicit the patient's history can lead to an incorrect presumption of IPF. A case series by Morell et al. [10] documented that 20 of 46 patients (43\%) diagnosed with IPF based on the 2011 American Thoracic Society (ATS) and European Respiratory Society (ERS) guidelines actually had chronic hypersensitivity pneumonitis upon re-evaluation.

The restricted approval by regulatory agencies of pirfenidone and nintedanib as therapeutic anti-fibrotic agents for IPF may prompt off-label use for other fibrotic lung diseases. Physicians may be swayed to mislabel a diagnosis of IPF in an attempt to get prescription coverage for these drugs for non-IPF fibrotic lung diseases. Thus, diagnostic codes recorded by physicians may not reflect an accurate diagnosis of the fibrotic lung disease.

\section{Environmental and genetic predisposing factors may be necessary for the development of the pulmonary fibrosis that we currently call "idiopathic" pulmonary fibrosis}

The lungs are constantly exposed to the ambient environment, which contains a variety of particulates, microbes, noxious fumes and pollutants. These exposures can occur in and outside homes, in the workplace, or elsewhere. This interaction creates a continuous cycle of lung injury, wound healing and tissue repair. The current understanding of IPF pathobiology highlights the importance of inhalational exposures as a precipitant of lung remodelling and fibrosis (figure 1). Support for this mechanistic pathway comes from comparisons to other environmental ILDs, which share similar genetic predispositions and histopathology.

In both IPF and other ILDs, disease does not develop in everyone who is exposed to the same environmental factors. A genetic predisposition is evident from families with multiple members affected. Studies of both familial and sporadic cases of IPF have shown increased susceptibility with variation in genes associated with telomere length (TERT, TERC, PARN, RTEL1), cellular adhesion and integrity (DSP, $A K A P 13, C T N N A, D P P 9)$, and the promoter region of the gene encoding mucin 5B (MUC5B) $[4,7,11]$. Recent observations suggest that some of these same genetic loci may be associated with an increased manifestation of chronic hypersensitivity pneumonitis [11]. Mice with silica-induced lung injury also display a transient increase in telomerase reverse transcriptase (TERT) [12]. The commonalities among these genetic pathways across a spectrum of fibrosing ILDs suggest that similar underlying pathobiologic pathways exist among different ILDs. 


\section{Genetically predisposed host}

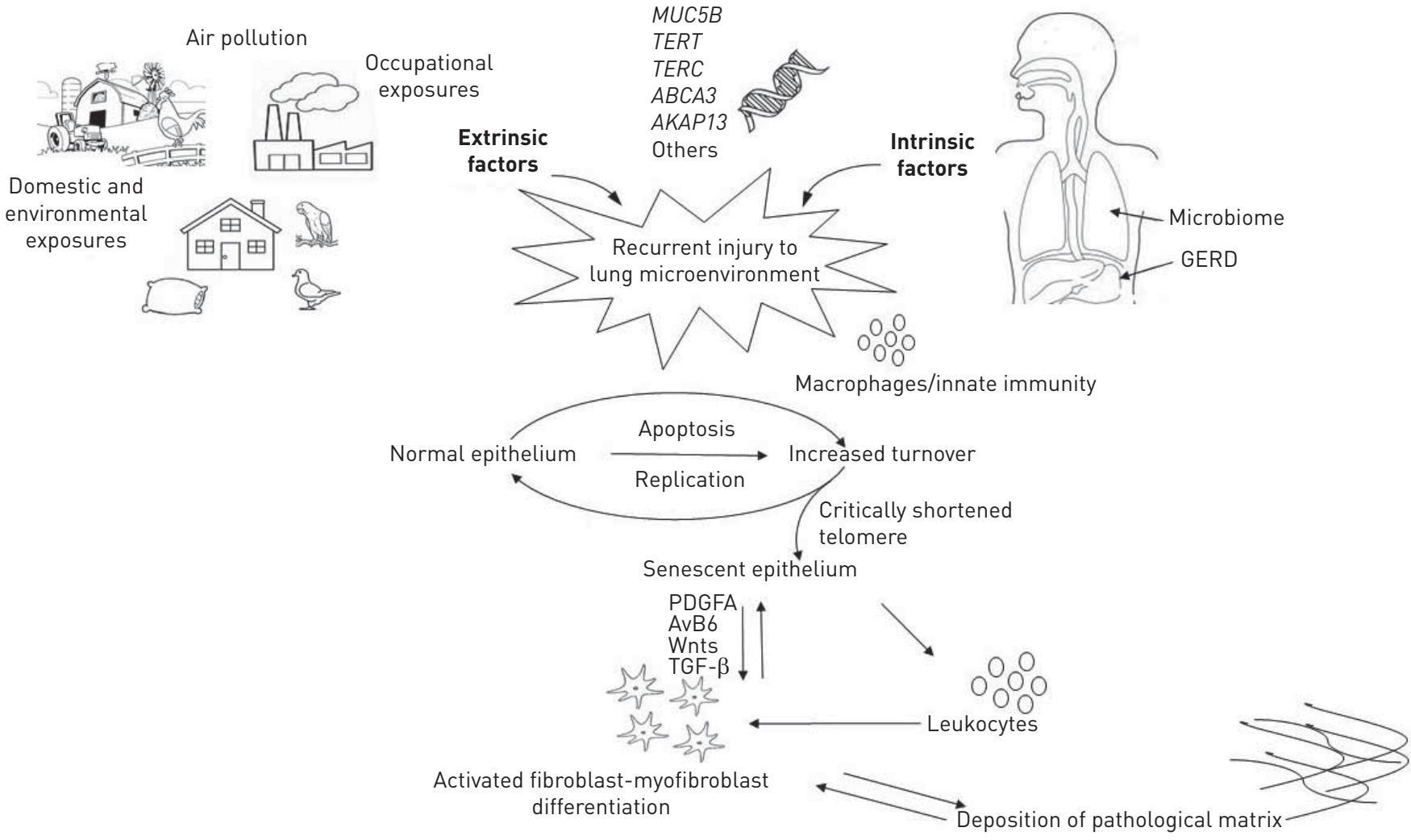

FIGURE 1 Proposed model for the pathogenesis of idiopathic pulmonary fibrosis. Extrinsic and intrinsic environmental exposures to the lung microenvironment cause recurrent airway injury. In the genetically predisposed host (mutations in mucin 5B (MUC5B), telomerase reverse transcriptase (TERT), telomerase RNA component (TERC), ATP binding cassette sub-family A3 (ABCA3), A-kinase anchor protein 13 (AKAP13), etc.), this cycle of increased epithelial turnover eventually leads to reprogramming to senescent epithelium. Fibroblasts are activated via pro-fibrotic mediators (platelet-derived growth factor subunit A (PDGFA), activating integrin B6 (avB6), Wnts, transforming growth factor- $\beta$ (TGF- $\beta$ )) that are released directly from abnormal epithelium, the activated innate immune system and leukocytes. Once activated, fibroblasts deposit pathological matrix, which leads to myofibroblast differentiation and progressive fibrosis. GERD: gastro-oesophageal reflux disease. Reproduced from [4] with permission.

In the current conception of IPF pathophysiology, the fibrogenic pathway is activated by recurrent airway injury leading to disordered repair of the epithelium. Oxidative stress or direct damage from air pollution exposure could potentially prime the epithelium to be more susceptible to injury from other exposures [13]. In genetically predisposed individuals, even minimal exposures to environmental agents, which include avian antigens and moulds, may be sufficient to cause disease.

Pathologic alteration of the epithelium is also more common with ageing, with telomere attrition contributing significantly to alveolar cell senescence [4]. Dead or dying epithelial cells stimulate the innate immune system, which plays a central role in wound healing. Whereas regulatory macrophages promote resolution of fibrosis through suppressive cytokines, M2 macrophages can secrete large amounts of pro-fibrotic cytokines and growth factors, such as fibroblast growth factor 2, platelet-derived growth factor, C-C motif chemokine ligand 18 and galectin-3 [14]. The abnormal epithelium activates mesenchymal cells either directly or through lymphocyte mediators, resulting in aberrant collagen production, extracellular matrix deposition, architectural distortion and the development of fibroblastic foci [4]. Once the cascade of fibrotic events has begun, it continues to self-perpetuate with progressive lung remodelling.

A similar process of airway injury, inflammation and fibrosis is observed in many of the other ILDs. In hypersensitivity pneumonitis, exposure to a range of exogenous inhaled particulates can cause an immunologic reaction with characteristic lymphocytic and granulomatous inflammation. In the setting of the pneumoconioses, such as asbestosis, the inhalation, deposition and retention of fibrogenic dusts is necessary for specific disease manifestation [15]. Disease does not develop in the majority of individuals who are exposed, but is dependent on multiple factors, including the dosage of particles inhaled, co-exposures and genetic susceptibility. Asbestosis and IPF share a similar distribution of disease as well as the characteristic UIP histologic pattern. Although asbestosis is distinguished by the appearance of the 
causative asbestos bodies, the other pathological features are similar, including epithelial cell hyperplasia, basement membrane denudation, alveolar consolidation and fibrogenic foci that are spatially and temporally heterogeneous [16].

Lung tissue specimens from patients otherwise diagnosed as having IPF demonstrate a bioaccumulation of inorganic particles. Several studies have used polarised light microscopy, electron microscopy and X-ray fluoroscopy to examine the mineral content of lung parenchyma and mediastinal lymph nodes [17-21]. Even after excluding cases with potential occupational exposures, these studies showed higher levels of retained aluminium, silica and nickel in individuals with IPF compared to controls with other causes of ILD. While these data do not prove that the dust particles are involved in the pathophysiology of the disease, they do suggest that even occult exposures could be an important component of disease manifestation.

\section{Intrinsic environmental factors}

Gastro-oesophageal reflux

Gastro-oesophageal reflux, and consequent microaspiration, is a common comorbidity in individuals with IPF, with a suggested prevalence of $>80 \%$ using oesophageal $\mathrm{pH}$ monitoring and manometry for diagnosis $[22,23]$. Many patients have "silent reflux" and are asymptomatic, with no complaints of heartburn or with nonacidic reflux identified on oesophageal studies. While some controversy still exists, gastro-oesophageal reflux has been proposed as a cause of disease development and progression [24, 25]. It is unclear whether the acid or other constituents of gastric fluid, e.g. bile salts, pepsin, food particles or bacteria, are pathogenic [26].

The optimal strategy for diagnosing and treating abnormal gastro-oesophageal reflux in individuals with IPF is uncertain. Evidence from observational studies suggests that antacid therapy in individuals with IPF is associated with less lung function decline and improved survival [27, 28]. The 2015 ATS/ERS/Japanese Respiratory Society/Latin American Thoracic Society international guideline committee on the treatment of IPF made a conditional recommendation for antacid treatment in all patients with IPF [1]. Surgical treatment of hiatal hernia using Nissen fundoplication is also associated with the stabilisation of oxygenation levels and a decreased rate of FVC decline in patients with IPF [29, 30]. A recent phase 2 randomised clinical trial investigating the therapeutic benefits of surgical correction of gastro-oesophageal reflux disease (GERD) suggests potential benefits associated with laparoscopic Nissen fundoplication, and warrants further studies to determine safety and efficacy [31].

\section{The lung microbiota}

The lung microbiota, including bacteria, fungi, viruses and bacteriophages, has also become an area of recent scientific interest and a potential therapeutic target in IPF. Bacteria and viruses can cause direct injury to airway epithelial cells and indirect damage by activating the host immune response to infection [32]. The apparent importance of an intact host defence was suggested in the PANTHER-IPF (Prednisone, Azathioprine, and $\mathrm{N}$-acetylcysteine: a Study that Evaluates Response in IPF) study, which showed worse outcomes in patients with IPF who were treated with immunosuppressants [33].

Viral infections, particularly human herpes virus and adenovirus, are thought to act as either exacerbating agents or initiators of disease. An increased incidence of human herpes viruses, such as cytomegalovirus, is seen in the lung samples of patients with IPF, acute IPF exacerbations and asymptomatic individuals who are at risk for familial IPF [34]. Many patients report a viral-type prodrome before the onset of disease symptoms [33]. These observations have led to the hypothesis that reactivation of latent viral infections may cause reprogramming of lung epithelial cells to produce fibrotic factors.

Studies demonstrate that individuals with IPF have a microbiome populated with a higher bacterial burden with more pathological strains. The aetiology of these changes to the microbial community is unknown, but may be due to recurrent gut microaspiration or a defective immune defence, or a consequence of the distorted parenchymal architecture from fibrosis [32]. Han et al. [35] found that Prevotella, Veillonella and Cronobacter species were the most prevalent species in a large cohort of individuals with IPF. After adjusting for confounders, they also found that the presence of Streptococcus and Staphylococcus species was associated with increased progression of disease. Although there was no group for comparison, the findings of this study are intriguing. Research is ongoing to determine whether chronic antibiotic administration can alter the lung microbiome and potentially affect disease progression [36].

\section{Extrinsic environmental factors/exposures \\ Tobacco smoke}

Tobacco exposure was one of the earliest and most consistently identified risk factors for IPF, with disease occurring more frequently in smokers. The association is particularly robust in individuals with familial IPF, 
with smokers (former or current) having an odds ratio of 3.6 (95\% CI 1.3-9.8) of developing disease [37]. Non-smokers have also been shown to have slower disease progression than either current or former smokers [38]. One cohort study suggested that current smokers may have a better prognosis than former smokers, but this was likely attributable to lead time bias from an earlier diagnosis rather than a true biologic mechanism [39].

\section{Exposures at the workplace loccult occupational exposures)}

The first epidemiologic evidence of the association between occupational exposures and IPF came from a case series that documented disease in certain professions with high amounts of dust and fume exposure, including industrial car cleaners, dairy farmers and welders [40]. Subsequent studies in the UK showed a higher incidence of IPF in industrialised areas and among men, who are more likely to have occupational exposures then women [41]. Subsequently there have been numerous case-control and cohort studies from diverse geographic areas, including the USA, Japan, Egypt and Korea, which have demonstrated similar associations. The most consistent data are for metal and wood dust exposure, with several studies also finding a dose-response relationship. Other occupational exposures and job activities that have been implicated include raising birds, hairdressing, stone cutting/polishing and exposure to livestock and vegetable dusts [42-46].

\section{Occult domestic and environmental exposures}

Many of the occupational exposures implicated above can also be present in domestic or recreational exposures of individuals with IPF. In particular, careful attention to both overt and occult exposure to organic dusts, unkempt ventilation, heating and cooling systems, moulds and avian antigens is warranted. A recent, provocative single-centre cohort study of individuals diagnosed with IPF at multidisciplinary discussion showed that individuals with pre-specified exposure to birds or moulds had a better survival rate compared to individuals without these pre-specified exposures [47]. In this study, a diagnosis of chronic hypersensitivity pneumonitis was excluded based on imaging features, bronchoalveolar lavage and presence of a specific IgG against exposed inhalants. This observation needs to be replicated and further explored. It is unclear whether patients with IPF and identifiable environmental exposures have a different natural history compared to individuals without these risk factors, or whether exposure remediation slowed the disease course.

\section{Air pollution}

Several recent studies highlight the importance of ambient air pollution in both the incidence and progression of IPF. Pollutants are a well-established cause of epithelial damage, oxidative stress and airway inflammation. There is also evidence that air pollution can precipitate epigenetic changes in the lung that enhance the pathogenicity of co-exposure to other antigens [13]. Through these mechanisms, air pollutants may initiate or contribute to alveolar damage and disordered repair. Three cohort studies in New Zealand, USA and France have shown that short-term exposure to pollutants is associated with accelerated decline in lung function and IPF exacerbations, while another recent study found that higher pollution exposure was associated with lower lung function [48-51]. A multi-city, population-based cohort study demonstrated that higher chronic exposure to air pollutants was associated with progression of subclinical ILD measurements on serial computed tomography scans [52]. In another multi-centre prospective study, an increase in the level of particles with a $50 \%$ cut-off aerodynamic diameter of $2.5 \mu \mathrm{m}$ was associated with an increased risk of developing hypersensitivity pneumonitis in urban cities in India [53].

\section{Clinical implications}

While evidence supports the hypothesis that environmental exposures contribute to the manifestation and progression of IPF, the implications for clinical practice need to be further delineated. Research is needed to determine effective means of identifying pertinent exposures and how this can help refine our definition of IPF, and to investigate whether there are any therapeutic benefits to the elimination of these exposures. While the distortion in lung architecture that results from fibrosis may be irreversible, removing precipitants of ongoing airway injury may help to prevent further damage.

Determining the relevant exposures in pulmonary fibrosis is challenging. In part, this is due to the presumed long latency period before disease manifestation. IPF is usually diagnosed in older adults after a lifetime of chronic and mixed exposures, making it difficult for both physicians and researchers to isolate potentially relevant causes. Most epidemiologic studies have used participant questionnaires, which raises the concern that recall bias could increase the likelihood of finding a positive association. In the clinical realm, appointment time constraints, patient unawareness and complex exposure histories are all barriers to obtaining a comprehensive environmental and occupational history. 
Standardised and detailed questionnaires designed according to local customs, cultural and geographical lifestyle and occupations could potentially address this gap. For example, a recent prospective cohort study of individuals with ILD in India identified relevant domestic exposures in $>40 \%$ of the participants. Interestingly, $47 \%$ of these were attributed to home air coolers, which are believed to be a source of mould and pigeon antigen exposure. Most of these individuals were diagnosed with hypersensitivity pneumonitis rather than IPF; this was predominantly based on imaging characteristics (which can mimic UIP) and the exposure history [54].

In addition to detailed questionnaires, there is a need for better objective measurements of exposure. Having a trained professional, e.g. an industrial hygienist, assess the home and work environment could better identify and quantify exposures. Individual circulating or other biomarkers of exposure can help determine the internalised dose and sensitisation. Because many of these exposures are ubiquitous in the environment, it will be important to determine whether there is individualised susceptibility to certain exposures.

Better questionnaires and objective assessments of environmental exposures may also help to distinguish individuals with other fibrotic lung diseases. In particular, chronic exposure to occult environmental factors can cause chronic hypersensitivity pneumonitis with UIP features. Such patients could easily be diagnosed with IPF if a careful and thorough exposure history is not obtained. Based on experience within our own institution and on observations by MoReLl et al. [10], $\geqslant 25 \%$ of patients referred for management of IPF may actually have chronic hypersensitivity pneumonitis or other fibrotic lung diseases.

The benefits of eliminating exposures need to be explored as a therapeutic approach. As outlined above, treatment of GERD may slow progression and perhaps improve survival in IPF. However, there is a paucity of data investigating similar outcomes for other environmental exposures. Measurements made during home and workplace assessments by a trained professional could lead to environmental remediation and potential health improvement. The efficacy of such interventions in reducing respiratory symptoms has been demonstrated in asthma [55]. No studies have been performed in individuals with IPF. We believe that avoiding intrinsic and extrinsic exposures that are known to cause lung injury, inflammation and fibrosis may minimise further insults to the fibrotic lung and could slow down disease progression. Hence, eliminating these environmental exposures in a patient with IPF could be an adjunct, cost-effective "treatment strategy" to approved pharmacologic modalities and the current standard of care.

\section{Conclusions}

We suggest that it is time to incorporate accumulating scientific evidence of the interaction between environmental exposures and genetic susceptibility into clinical practice. Research should focus on "environmental factors", i.e. those "intrinsic" and "extrinsic" factors that may play important roles in the pathogenesis of IPF, especially in genetically predisposed individuals living in polluted urban areas. There is a critical need for more research to characterise relevant exposures in IPF patients through the development of standardised questionnaires, identification and validation of circulating biomarkers, and investigations by industrial hygienists. Work is needed to understand the ecogenetics of disease pathobiology and how gene-environment interactions impact disease development and progression. Finally, there is an absolute need for clinical studies to determine whether the elimination of both intrinsic and extrinsic environmental factors can have an impact on the prognosis of patients diagnosed with IPF based on current understanding, guidelines and standard of care.

In essence, we hope that our perspective will increase awareness of occult intrinsic and extrinsic environmental factors that may be contributing to the pathogenesis of "idiopathic" pulmonary fibrosis and prompt well-designed investigations of these factors in future studies. We believe that such studies will 1) demonstrate that the vast majority of patients with "IPF" have occult exposures known to induce lung injury and/or perpetuate progression of pulmonary fibrosis; 2) provide evidence that avoiding these exposures can help prevent further injury to the lung microenvironment, particularly in genetically susceptible individuals and in patients with "early UIP/IPF"; and 3) eventually reduce the incidence of true IPF over time.

Author contributions: G. Raghu initiated the concept of writing this perspective. C. Sack and G. Raghu contributed equally to researching, collating and writing this paper.

Conflict of interest: C. Sack has nothing to disclose. G. Raghu reports research grants for IPF studies from National Institutes of Health during the conduct of the study; has acted as a consultant on IPF studies for Biogen, BMS, Fibrogen, Gilead Sciences, Promedior, Roche-Genentech, Bellerophan and Nitto, has received personal fees, non-financial support and has acted as a consultant for Boehringer-Ingelheim and Sanofi, and has received personal fees and has acted as a consultant for Patara and Veracyte, outside the submitted work. 


\section{References}

1 Raghu G, Rochwerg B, Zhang Y, et al. An Official ATS/ERS/JRS/ALAT clinical practice guideline: treatment of idiopathic pulmonary fibrosis. An update of the 2011 clinical practice guideline. Am J Respir Crit Care Med 2015; 192: e3-e19.

2 Raghu G, Collard HR, Egan JJ, et al. An official ATS/ERS/JRS/ALAT statement: idiopathic pulmonary fibrosis: evidence-based guidelines for diagnosis and management. Am J Respir Crit Care Med 2011; 183: 788-824.

3 Raghu G, Remy-Jardin M, Myers JL, et al. Diagnosis of idiopathic pulmonary fibrosis. An official ATS/ERS/JRS/ ALAT clinical practice guideline. Am J Respir Crit Care Med 2018; 198: e44-e68.

4 Wolters PJ, Blackwell TS, Eickelberg O, et al. Time for a change: is idiopathic pulmonary fibrosis still idiopathic and only fibrotic? Lancet Respir Med 2018; 6: 154-160.

5 Wells AU, Brown KK, Flaherty KR, et al. What's in a name? That which we call IPF, by any other name would act the same. Eur Respir J 2018; 51: 1800692.

6 American Thoracic Society. Idiopathic pulmonary fibrosis: diagnosis and treatment. International consensus statement. American Thoracic Society (ATS), and the European Respiratory Society (ERS). Am J Respir Crit Care Med 2000; 161: 646-664.

7 Lederer DJ, Martinez FJ. Idiopathic pulmonary fibrosis. N Engl J Med 2018; 378: 1811-1823.

8 Lynch DA, Sverzellati N, Travis WD, et al. Diagnostic criteria for idiopathic pulmonary fibrosis: a Fleischner Society white paper. Lancet Respir Med 2018; 6: 138-153.

9 Collins BF, McClelland RL, Ho LA, et al. Sarcoidosis and IPF in the same patient-a coincidence, an association or a phenotype? Respir Med 2018; 144S: S20-S27.

10 Morell F, Villar A, Montero M-Á, et al. Chronic hypersensitivity pneumonitis in patients diagnosed with idiopathic pulmonary fibrosis: a prospective case-cohort study. Lancet Respir Med 2013; 1: 685-694.

11 Ley B, Newton CA, Arnould I, et al. The MUC5B promoter polymorphism and telomere length in patients with chronic hypersensitivity pneumonitis: an observational cohort-control study. Lancet Respir Med 2017; 5: 639-647.

12 Tashiro J, Rubio GA, Limper AH, et al. Exploring animal models that resemble idiopathic pulmonary fibrosis. Front Med 2017; 4: 118.

13 Mookherjee N, Piyadasa H, Ryu MH, et al. Inhaled diesel exhaust alters the allergen-induced bronchial secretome in humans. Eur Respir J 2018; 51: 1701385.

14 Ahluwalia N, Shea BS, Tager AM. New therapeutic targets in idiopathic pulmonary fibrosis. Aiming to rein in runaway wound-healing responses. Am J Respir Crit Care Med 2014; 190: 867-878.

15 Litow FK, Petsonk EL, Bohnker BK, et al. Occupational interstitial lung diseases. J Occup Environ Med 2015; 57: $1250-1254$.

16 Moore BB, Lawson WE, Oury TD, et al. Animal models of fibrotic lung disease. Am J Respir Cell Mol Biol 2013; 49: $167-179$.

17 Tsuchiya $\mathrm{K}$, Inase $\mathrm{N}$, Ichinose $\mathrm{S}$, et al. Elemental analysis of inorganic dusts in lung tissues of interstitial pneumonias. J Med Dent Sci 2007; 54: 9-16.

18 Hashimoto $\mathrm{H}$, Tajima H, Mizoguchi I, et al. [Elemental analysis of hilar and mediastinal lymph nodes in idiopathic pulmonary fibrosis]. Nihon Kyobu Shikkan Gakkai Zasshi 1992; 30: 2061-2068.

19 Inoue M. [Quantitative and qualitative analyses of inorganic dusts in idiopathic interstitial pneumonia (IIP)]. Hokkaido Igaku Zasshi 1986; 61: 745-754.

20 Monsó E, Tura JM, Pujadas J, et al. Lung dust content in idiopathic pulmonary fibrosis: a study with scanning electron microscopy and energy dispersive X ray analysis. Br J Ind Med 1991; 48: 327-331.

21 Raghu G, Collins BF, Xia D, et al. Pulmonary fibrosis associated with aluminum trihydrate (Corian) dust. $N$ Engl $J$ Med 2014; 370: 2154-2156.

22 Tobin RW, Pope CE, Pellegrini CA, et al. Increased prevalence of gastroesophageal reflux in patients with idiopathic pulmonary fibrosis. Am J Respir Crit Care Med 1998; 158: 1804-1808.

23 Raghu G, Freudenberger TD, Yang S, et al. High prevalence of abnormal acid gastro-oesophageal reflux in idiopathic pulmonary fibrosis. Eur Respir J 2006; 27: 136-142.

24 Raghu G, Meyer KC. Silent gastro-oesophageal reflux and microaspiration in IPF: mounting evidence for anti-reflux therapy? Eur Respir J 2012; 39: 242-245.

25 Ghebre YT, Raghu G. Idiopathic pulmonary fibrosis: novel concepts of proton pump inhibitors as antifibrotic drugs. Am J Respir Crit Care Med 2016; 193: 1345-1352.

26 Johannson KA, Strâmbu I, Ravaglia C, et al. Antacid therapy in idiopathic pulmonary fibrosis: more questions than answers? Lancet Respir Med 2017; 5: 591-598.

27 Lee JS, Collard HR, Anstrom KJ, et al. Anti-acid treatment and disease progression in idiopathic pulmonary fibrosis: an analysis of data from three randomised controlled trials. Lancet Respir Med 2013; 1: 369-376.

28 Lee JS, Ryu JH, Elicker BM, et al. Gastroesophageal reflux therapy is associated with longer survival in patients with idiopathic pulmonary fibrosis. Am J Respir Crit Care Med 2011; 184: 1390-1394.

29 Linden PA, Gilbert RJ, Yeap BY, et al. Laparoscopic fundoplication in patients with end-stage lung disease awaiting transplantation. J Thorac Cardiovasc Surg 2006; 131: 438-446.

30 Raghu G, Morrow E, Collins BF, et al. Laparoscopic anti-reflux surgery for idiopathic pulmonary fibrosis at a single centre. Eur Respir J 2016; 48: 826-832.

31 Raghu G, Pellegrini CA, Yow E, et al. Laparoscopic anti-reflux surgery for the treatment of idiopathic pulmonary fibrosis (WRAP-IPF): a multicentre, randomised, controlled phase 2 trial. Lancet Respir Med 2018; 6: 707-714.

32 Fastrès A, Felice F, Roels E, et al. The lung microbiome in idiopathic pulmonary fibrosis: a promising approach for targeted therapies. Int J Mol Sci 2017; 18: 2735.

33 Moore BB, Moore TA. Viruses in idiopathic pulmonary fibrosis. Etiology and exacerbation. Ann Am Thorac Soc 2015; 12: Suppl. 2, S186-S192.

34 Molyneaux PL, Maher TM. The role of infection in the pathogenesis of idiopathic pulmonary fibrosis. Eur Respir Rev 2013; 22: 376-381.

35 Han MK, Zhou Y, Murray S, et al. Lung microbiome and disease progression in idiopathic pulmonary fibrosis: an analysis of the COMET study. Lancet Respir Med 2014; 2: 548-556.

36 CleanUP IPF for the Pulmonary Trials Cooperative. https:/clinicaltrials.gov/ct2/show/NCT02759120 Date last accessed: September 3, 2018 

Respir Crit Care Med 2005; 172: 1146-1152. Antoniou KM, Hansell DM, Rubens MB, et al. Idiopathic pulmonary fibrosis. Am J Respir Crit Care Med 2008; 177: 190-194.

39 King TE, Schwarz MI, Brown K, et al. Idiopathic pulmonary fibrosis. Am J Respir Crit Care Med 2001; 164: 1025-1032.

40 Taskar V, Coultas D. Exposures and idiopathic lung disease. Semin Respir Crit Care Med 2008; 29 : 670-679.

41 Scott J, Johnston I, Britton J. What causes cryptogenic fibrosing alveolitis? A case-control study of environmental exposure to dust. BMJ 1990; 301: 1015-1017.

42 Awadalla NJ, Hegazy A, Elmetwally RA, et al. Occupational and environmental risk factors for idiopathic pulmonary fibrosis in Egypt: a multicenter case-control study. Int J Occup Environ Med 2012; 3: 107-116.

43 Baumgartner KB, Samet JM, Coultas DB, et al. Occupational and environmental risk factors for idiopathic pulmonary fibrosis: a multicenter case-control study. Collaborating Centers. Am J Epidemiol 2000; 152: 307-315.

44 Iwai K, Mori T, Yamada N, et al. Idiopathic pulmonary fibrosis. Epidemiologic approaches to occupational exposure. Am J Respir Crit Care Med 1994; 150: 670-675.

45 Gustavsson P, Jakobsson R, Nyberg F, et al. Occupational exposure and lung cancer risk: a population-based casereferent study in Sweden. Am J Epidemiol 2000; 152: 32-40.

46 Ekström M, Gustafson T, Boman K, et al. Effects of smoking, gender and occupational exposure on the risk of severe pulmonary fibrosis: a population-based case-control study. BMJ Open 2014; 4: e004018.

47 De Sadeleer LJ, Verleden SE, De Dycker E, et al. Clinical behaviour of patients exposed to organic dust and diagnosed with idiopathic pulmonary fibrosis. Respirology 2018; 23: 1160-1165.

48 Winterbottom CJ, Shah RJ, Patterson KC, et al. Exposure to ambient particulate matter is associated with accelerated functional decline in idiopathic pulmonary fibrosis. Chest 2017; 153: 1221-1228.

49 Sesé L, Nunes H, Cottin V, et al. Role of atmospheric pollution on the natural history of idiopathic pulmonary fibrosis. Thorax 2018; 73: 145-150.

50 Johannson KA, Vittinghoff E, Lee K, et al. Acute exacerbation of idiopathic pulmonary fibrosis associated with air pollution exposure. Eur Respir J 2014; 43: 1124-1131.

51 Johannson KA, Vittinghoff E, Morisset J, et al. Air pollution exposure is associated with lower lung function, but not changes in lung function, in patients with idiopathic pulmonary fibrosis. Chest 2018; 154: 119-125.

52 Sack C, Vedal S, Sheppard L, et al. Air pollution and subclinical interstitial lung disease: the Multi-Ethnic Study of Atherosclerosis (MESA) air-lung study. Eur Respir J 2017; 50: 1700559.

53 Singh S, Collins BF, Bairwa M, et al. Hypersensitivity pneumonitis and its correlation with ambient air pollution in urban India. Eur Respir J 2019; 53: 1801563.

54 Singh S, Collins BF, Sharma BB, et al. Interstitial lung disease in India. Results of a prospective registry. Am $J$ Respir Crit Care Med 2017; 195: 801-813.

55 Le Cann P, Paulus H, Glorennec P, et al. Home environmental interventions for the prevention or control of allergic and respiratory diseases: what really works. J Allergy Clin Immunol Pract 2017; 5: 66-79. 